\section{Polimorfismos de los genes del sistema leptina- melanocortina asociados con la obesidad en la población adulta de Barranquilla}

Pilar Garavito ${ }^{1}$ María Isabel Mosquera-Heredia'1, Luis Fang ${ }^{1}$, Fausto Payares ${ }^{1}$, Marta Ruiz ${ }^{1}$, Isis Arias ${ }^{1}$, Rafael Tuesca², Édgar Navarro², Carlos Silvera-Redondo ${ }^{1}$

${ }^{1}$ Grupo de Investigación Genética y Medicina Molecular, Departamento de Medicina, Universidad del Norte, Barranquilla, Colombia

${ }^{2}$ Grupo de Investigación Proyecto UNI, Departamento de Salud Pública, Universidad del Norte, Barranquilla, Colombia

Introducción. La obesidad se considera un grave problema de salud pública y por ello se hacen esfuerzos en la búsqueda de genes como el $L E P$, el $L E P R$ y el $M C 4 R$ del sistema leptina-melanocortina, el cual opera en la regulación neuroendocrina de la ingestión y el equilibrio energético e influye en la patogenia de la enfermedad. Los resultados contradictorios en torno a la asociación de estos genes con la obesidad plantean la necesidad de nuevas investigaciones.

Objetivo. Analizar los polimorfismos rs2167270 del gen LEP, rs1137101 del gen LEPR y rs17782313 del gen MC4R asociados con la obesidad y sus variables clínicas y bioquímicas en una muestra de pacientes adultos de Barranquilla.

Materiales y métodos. Se estudiaron 111 personas obesas y 155 no obesas como controles. Los polimorfismos se determinaron mediante reacción en cadena de la polimerasa (PCR) en tiempo real. Se tomaron las medidas antropométricas, se evaluó la presión arterial y se hicieron pruebas bioquímicas.

Resultados. No se encontraron diferencias estadísticas en la frecuencia alélica y genotípica de los polimorfismos en los grupos estudiados. En cuanto a las variables clínicas y bioquímicas, el genotipo CC del polimorfismo rs17782313 del gen MC4R, se asoció con un aumento de la presión arterial sistólica y, el alelo T y su genotipo homocigoto, con una disminución del colesterol HDL en los obesos. No se evidenció ningún efecto de los otros polimorfismos en estas variables.

Conclusiones Los polimorfismos rs2167270 del gen LEP, rs1137101 del gen LEPR y rs17782313 del gen $M C 4 R$, no se asociaron con obesidad en la población analizada. Se encontró que el polimorfismo rs17782313 del gen MC4R influyó en el aumento de la presión arterial sistólica y la disminución del colesterol HDL en las personas obesas.

Palabras clave: obesidad/genética; polimorfismo genético.

Polymorphisms of leptin-melanocortin system genes associated with obesity in an adult population from Barranquilla

Introduction: Obesity is considered a serious public health problem. Efforts have been directed to search for candidate genes such as $L E P, L E P R$, and MC4R involved in the leptinmelanocortin system. The neuroendocrine regulation of these genes on energy intake and balance influences the pathogenesis of this disease. Contradictory results regarding the association of these genes with obesity raise the need for new research.

Objective: To analyze the association between obesity and LEP rs2167270, LEPR rs1137101, and MC4R rs17782313 polymorphisms and the clinical and biochemical variables in obese adults from Barranquilla, Colombia.

Materials and methods: We analyzed 111 obese adults and 155 non-obese individuals as controls. The polymorphisms were determined by real-time PCR. Besides, anthropometric measures, blood pressure, and biochemical tests were evaluated.

Results: No statistical differences were found in allele and genotype frequencies of gene polymorphisms between groups. The CC genotype of $M C 4 R$ rs 17782313 polymorphism was associated with increased systolic blood pressure and T allele and TT genotype, with decreased HDL cholesterol in obese adults. The effect of the other polymorphisms on these variables was not evidenced.

Conclusions: LEP rs2167270, LEPR rs 1137101, and MC4R rs 17782313 polymorphisms were not associated with obesity in the population under study. MC4R rs 17782313 polymorphisms were associated with an increase in systolic blood pressure and a decrease in HDL cholesterol.

Keywords: Obesity/genetics; polymorphism, genetic.
Conflicto de intereses:

Los autores declaran que no existen conflictos de intereses. 
La obesidad se considera un grave problema de salud pública a nivel mundial, ya que constituye el principal tipo de malnutrición del adulto. Según la Organización Mundial de la Salud (OMS), en el 2016, más de 1.900 millones de adultos tenían exceso de peso, lo que representa el $39 \%$ de la población, y el $13 \%$ de ellos se consideraban obesos (1).

El gran impacto de la obesidad en la morbilidad y la calidad de vida de los individuos se debe a que constituye un factor de riesgo de diabetes, cardiopatías isquémicas, enfermedades del aparato locomotor y algunos tipos de cáncer (1). Por lo tanto, la comprensión de su etiología y de la forma de regular el peso corporal tiene gran importancia científica, sanitaria y económica.

Un elemento clave esencial para comprender la etiología y la patogenia de la obesidad es el papel del sistema leptina-melanocortinas en el control del almacenamiento de la grasa corporal mediante la regulación coordinada de la conducta alimentaria, el metabolismo, las reacciones neuroendocrinas, el sistema nervioso autónomo y el balance de energía del cuerpo (2).

El gen LEP está situado en 7q32.1, mide $20 \mathrm{~kb}$ y está constituido por tres exones y dos intrones; codifica para la leptina, una hormona proteica constituida por 167 aminoácidos con peso molecular de $16 \mathrm{kDa}$, en tanto que su receptor, codificado por el gen $L E P R$, está constituido por 24 exones y se localiza en 1p31.3. Por su parte, el gen $M C 4 R$ se ubica en 18q22, solo tiene un exon y codifica para el receptor 4 de melanocortina.

La leptina es secretada fundamentalmente por el tejido adiposo blanco. Sus concentraciones circulantes son proporcionales al contenido de la grasa corporal (3). Esta hormona atraviesa la barrera hematoencefálica e interactúa con su receptor específico en el núcleo arcuato del hipotálamo y actúa como una señal indicadora de las reservas energéticas. Las POMC/ CART y las AGRP/NPY son las poblaciones neuronales del núcleo arcuato que expresan el receptor de la leptina en forma importante. Las primeras, las POMC/CART, conducen señales anorexigénicas mediante los derivados de la proopiomelanocortina (POMC), en tanto que las AGRP/NPY conducen señales orexigénicas mediante el neuropéptido Y (NPY) y y la proteína relacionada con agouti (Agouti-related protein, AgRP). La ausencia de leptina o la reducción de su concentración provocan una mayor ingestión alimentaria debido a la expresión de AGRP/NPY. En el caso contrario, se promueve la expresión de la POMC, la cual se escinde después de su traducción en péptidos llamados melanocortinas, que actuarían como ligandos endógenos del receptor 4 de melanocortina (MC4R), disminuyendo el apetito (4).

Cuando se reduce la capacidad de la leptina para regular el apetito y el aumento de peso, se desarrolla una resistencia contra ella que puede conducir a la obesidad. Algunos mecanismos implicados en dicha resistencia son los defectos en el transporte de la leptina a través de la barrera hematoencefálica, los defectos en la señalización del receptor de la leptina y las alteraciones en las vías nerviosas involucradas en la regulación de la homeostasis energética (5).

Se ha demostrado que algunos polimorfismos de un solo nucleótido (SNP) en los genes $L E P, L E P R$ y $M C 4 R$, se relacionan con el fenotipo y los marcadores metabólicos asociados con la obesidad. Se ha sugerido que el polimorfismo rs2167270 del gen LEP podría estar asociado con la variación en el aumento de la leptinemia y la propensión a la obesidad (6). Asimismo, el SNP rs1137101 del gen LEPR también se ha relacionado con un aumento 
en el índice de masa corporal (IMC), hiperleptinemia y predisposición a la resistencia a la leptina (7).

Por su parte, los metaanálisis de estudios de asociación en todo el genoma (Genome-Wide Association Study, GWAS) en caucásicos, revelaron que la variante rs 17782313 del gen MC4R tiene una fuerte asociación con valores elevados del IMC (8) y con la aparición temprana de obesidad grave (9). Estas asociaciones se han replicado en múltiples poblaciones, incluidos niños, adolescentes y adultos. Sin embargo, se han reportado resultados contradictorios alrededor del mundo, lo cual indica la necesidad de adelantar nuevas investigaciones al respecto.

El objetivo del presente estudio fue analizar los polimorfismos rs2167270 del gen $L E P$, rs 1137101 del gen $L E P R$ y rs 17782313 del gen $M C 4 R$ asociados con la obesidad en una muestra de adultos de Barranquilla.

\section{Materiales y métodos}

\section{Población y muestra}

Se hizo un estudio descriptivo transversal con análisis de casos y controles, lo que implicó hacer un muestreo poblacional en Barranquilla. Se compararon dos grupos en el rango de edad entre los 20 y los 69 años: los casos eran individuos con obesidad y un IMC de $30 \mathrm{~kg} / \mathrm{m}^{2}$ o mayor, y los sujetos de control tenían un IMC entre 18,5 y $24,9 \mathrm{~kg} / \mathrm{m}^{2}$.

La población analizada era una muestra de los participantes en el estudio "Salud global: estrategia de investigación aplicada para el estudio y la intervención del síndrome metabólico en Barranquilla". El proceso de selección y la técnica de recolección de datos se describen en la publicación de Giraldo-Castrillón, et al. (10).

Para estimar el tamaño de la muestra, se utilizó un porcentaje de $61 \%$ en los polimorfismos de los sistemas genéticos bajo estudio, tomando como referencia la frecuencia del polimorfismo del gen LEP (A19G) (11), un nivel de confianza del $95 \%$, un poder del $80 \%$ y una relación entre casos y controles de 1:1, lo que resultó en 116 participantes en el grupo de los casos y 116 en el de los controles.

Los criterios de exclusión incluyeron embarazo, hipotiroidismo, síndrome de Cushing, hipogonadismo o síndromes monogénicos relacionados con obesidad, así como estar en tratamiento para diabetes o dislipidemia.

Todos los participantes firmaron un consentimiento informado y el estudio se ajustó a los principios éticos de la investigación en seres humanos consignados en la Declaración de Helsinki y la Resolución 8430 de 1993 del Ministerio de Salud de Colombia. Además, el estudio contó con la aprobación del Comité de Ética de la Universidad del Norte.

\section{Parámetros clínicos y bioquímicos}

Los parámetros antropométricos de peso, talla, cintura y cadera se tomaron siguiendo los protocolos estandarizados. El IMC se calculó con la fórmula de peso $(\mathrm{kg}) / \mathrm{talla}\left(\mathrm{m}^{2}\right)$. Se clasificó como individuos con sobrepeso a aquellos con un IMC entre 25 y $29,9 \mathrm{~kg} / \mathrm{m}^{2}$, y con obesidad, a quienes tenían un IMC mayor o igual a $30 \mathrm{~kg} / \mathrm{m}^{2}$. Se consideró normal una circunferencia de cintura de menos de 88 centímetros en las mujeres y de menos de 102 centímetros 
en los hombres (12). Además, la presión arterial sistólica y la diastólica se midieron en dos momentos diferentes, siguiendo el protocolo de la OMS (13). Los puntos de corte se tomaron del Eighth Joint National Committee (JNC 8) para la prevención, detección, evaluación y tratamiento de la hipertensión (14).

A los individuos participantes se les extrajeron $10 \mathrm{ml}$ de sangre total mediante venopunción convencional después de 12 horas de ayuno; $5 \mathrm{ml}$ se recolectaron en un tubo con EDTA para los estudios genéticos y $5 \mathrm{ml}$ en un tubo seco para los estudios bioquímicos utilizando el sistema Vacutainer ${ }^{\mathrm{TM}}$. El colesterol total, los triglicéridos y la glucemia se determinaron con métodos enzimáticos fotocolorimétricos comerciales (Biosystems); el cHDL se separó al precipitar selectivamente las lipoproteínas de baja densidad (low density lipoprotein, LDL) y de muy baja densidad (very low density lipoprotein, VLDL) agregando ácido fosfotúngstico en presencia de iones de magnesio. El CLDL se calculó de forma indirecta empleando la ecuación de Friedewald (15). Los puntos de corte utilizados para la clasificación de la dislipidemia se determinaron a partir de las guías del Adult Treatment Panel III (ATP III) (16).

La insulina se determinó mediante quimioluminiscencia (Siemens Medical) y la hemoglobina glucosilada por colorimetria por afinidad al boronato (NycoCard Axis-Shield ${ }^{\mathrm{TM}}$ ) siguiendo las instrucciones de la casa comercial.

\section{Extracción del ADN genómico y genotipificación}

El ADN genómico se obtuvo usando el estuche comercial UltraClean Blood $^{\mathrm{TM}}$ (MO BIO Laboratories, Inc.), siguiendo las instrucciones recomendadas por el fabricante. La cuantificación del ADN se llevó a cabo en un espectrofotómetro NanoDrop $2000^{\mathrm{TM}}$ que, además de los valores de concentración, también aporta los valores de relación 260/280 (ADN/ proteínas) y 260/230 (ADN y solventes orgánicos) e información sobre la pureza del producto. Se diluyeron todas las muestras de ADN hasta llevarlas a una concentración de $20 \mathrm{ng} / \mu \mathrm{l}$ y se almacenaron a $-20 \stackrel{\circ}{ } \mathrm{C}$.

Los polimorfismos rs2167270 del gen LEP, rs1137101 del gen LEPR y rs17782313 del gen MC4R se determinaron mediante PCR en tiempo real con el método de discriminación alélica TaqMan PRISM 7500 Real-Time PCR Systems ${ }^{\text {TM }}$ (Applied Biosystems) con los ensayos ID C_15966471_20, ID C_8722581_10 y ID C_32667060_10, respectivamente.

La mezcla de amplificación para un volumen final de reacción de $25 \mu \mathrm{l}$ contenía 20 ng de ADN genómico, 12,5 $\mu$ l de TaqMan Universal PCR Master $\mathrm{Mix}^{\mathrm{TM}}$, mezcla que contiene polimerasa AmpliTaq Gold DNA ${ }^{\mathrm{TM}}$, AmpErase Uracil N-glicosilasa (UNG), desoxinucleótidos (dNTP) con desoxiuridina 5'-trifosfato (dUTP), referencia pasiva (ROX) y solución tampón, así como $1,25 \mu \mathrm{l}$ de 20X de mezcla para cada uno de los ensayos de genotipificación descritos anteriormente y específica para cada polimorfismo, con $18 \mu \mathrm{M}$ de cada cebador y $4 \mu \mathrm{M}$ de cada sonda (VIC/FAM).

Todas las pruebas se hicieron siguiendo un mismo protocolo de amplificación y detección que se iniciaba con un ciclo a $50 \stackrel{\circ}{ } \mathrm{C}$ durante 2 minutos, un ciclo a $95 \stackrel{\circ}{\circ}$ durante 10 minutos, 40 ciclos a $95 \stackrel{\circ}{\mathrm{C}}$ durante 15 segundos cada uno y un ciclo a $60{ }^{\circ} \mathrm{C}$ durante 90 segundos.

Por último, los genotipos se determinaron a partir del resultado de los productos de amplificación observados como curvas de amplificación reconocidas a partir de la marcación para cada sonda (VIC/FAM). 


\section{Análisis estadístico}

Para el análisis estadístico, se estimaron las frecuencias alélicas y genotípicas de los SNP estudiados en ambos grupos. Las frecuencias alélicas se usaron para estimar el equilibrio genético de Hardy-Weinberg. El análisis de asociación genética se estimó con el programa Arlequin, versión 3.5.

El análisis de asociación de genotipos se hizo mediante la prueba de ji al cuadrado de Pearson con corrección de Bonferroni para el ajuste del valor de $p$ en el programa estadístico SPSS ${ }^{\mathrm{TM}}$, versión 20 (IBM Corp., USA). También, se estimó el riesgo de obesidad asociado con cada genotipo y el alelo mediante el cálculo de la razón de momios (odds ratio, OR) y sus correspondientes intervalos de confianza del $95 \%$ usando modelos de regresión logística ajustados por sexo.

Las variables numéricas se expresaron como medianas y rangos intercuartílicos (RIC). Estas variables se compararon en los dos grupos de estudio y entre los polimorfismos mediante las pruebas estadísticas $U$ de Mann-Whitney y Kruskal-Wallis, respectivamente, debido a que no cumplieron con el supuesto de normalidad.

El contraste de las variables categóricas se hizo mediante la prueba de ji al cuadrado de Pearson. A estos datos se les determinó la corrección de Bonferroni del valor de p. La significación estadística se estableció en p<0,05.

\section{Resultados}

Se incluyeron y compararon dos grupos en el rango de edad entre los $20 \mathrm{y}$ los 69 años: fueron 111 casos con obesidad y un IMC de $30 \mathrm{~kg} / \mathrm{m}^{2}$ o mayor, y 155 de control con un IMC entre 18,5 y $24,9 \mathrm{~kg} / \mathrm{m}^{2}$.

Estas cantidades corresponden a una disminución del 4,3 \% (5 sujetos) en el número de casos y a un incremento de 39 (33,6\%) en el de controles, para evitar la disminución del poder del estudio. Los casos que no participaron abandonaron el estudio por temor a la venopunción y por no haber completado las pruebas bioquímicas.

La edad promedio fue de 40,8 $\pm 12,3$ años entre los casos y de $35 \pm 12,7$ años entre los controles. En ambos grupos predominó el sexo femenino, con porcentajes del 66,1 y $53,5 \%$, respectivamente.

En el cuadro 1 se presentan las características clínicas y bioquímicas de la población estudiada, así como las diferencias encontradas al comparar los grupos. La edad, las medidas antropométricas, la glucemia en ayunas, los triglicéridos, la $\mathrm{HbA} 1 \mathrm{C}$ y el riesgo cardiovascular global, fueron significativamente mayores en el grupo de los casos.

Las frecuencias alélicas y genotípicas de los polimorfismos estudiados, se presentan en el cuadro 2. Se observó que estas frecuencias se mantuvieron en equilibrio de Hardy-Weinberg en el grupo de control, excepto para el polimorfismo del gen MC4R. También, se presenta en este cuadro un análisis de asociación de estas frecuencias con la obesidad, el cual no evidenció diferencias estadísticas entre los grupos para ninguno de los SNP estudiados.

Además, se analizó la asociación de los polimorfismos con las medidas antropométricas, la presión arterial y las pruebas bioquímicas en los dos grupos de estudio y se encontró que el genotipo CC del polimorfismo 
rs17782313 del gen MC4R se asoció con un aumento de la presión arterial sistólica y, el alelo T y su genotipo homocigoto, con una disminución del cHDL, pero solo en el grupo de obesos. No se evidenció asociación de los otros polimorfismos con las variables estudiadas (cuadro 3 , figuras 1 y 2 ).

Cuadro 1. Características clínicas y bioquímicas de la población analizada

\begin{tabular}{|c|c|c|c|c|c|c|c|}
\hline \multirow{2}{*}{ Sexo } & \multicolumn{2}{|c|}{$\begin{array}{c}\text { Casos } \\
(n=111) n(\%)\end{array}$} & \multicolumn{2}{|c|}{$\begin{array}{c}\text { Control } \\
(n=155) n(\%)\end{array}$} & \multirow[t]{2}{*}{$\mathbf{p}$} & \multirow[t]{2}{*}{ OR } & \multirow[t]{2}{*}{$\mathrm{IC}_{95 \%}$} \\
\hline & & & & & & & \\
\hline Masculino & 41 & $(36,9)$ & 72 & $(46,5)$ & & 1 & --- \\
\hline Femenino & 70 & $(63,1)$ & 83 & $(53,5)$ & $0,155^{\dagger}$ & 1,48 & $0,9-2,4$ \\
\hline \multicolumn{8}{|l|}{ Edad (años) } \\
\hline Mediana [RIC] & 40 & (19) & 32 & {$[20]$} & $0,00^{\star *}$ & & \\
\hline \multicolumn{8}{|l|}{ Riesgo cardiovascular } \\
\hline Muy bajo & 11 & $(9,9)$ & 46 & $(29,7)$ & & 1 & --- \\
\hline Вајо & 21 & $(18,9)$ & 28 & $(18,1)$ & & 3,42 & $1,4-8,2$ \\
\hline Alto & 79 & $(71,2)$ & 81 & $(52,3)$ & $0,00^{*+}$ & 5,79 & $2,3-14,5$ \\
\hline \multicolumn{8}{|l|}{ Hipertensión } \\
\hline Normal $<120 /<80 \mathrm{~mm} \mathrm{Hg}$ & 58 & $(52,3)$ & 97 & $(62,6)$ & & 1 & --- \\
\hline Prehipertenso 120-139/80-89 mm Hg & 34 & $(30,6)$ & 41 & $(26,5)$ & & 1,3 & $0,7-2,4$ \\
\hline HTA-1 140-159/90-99 mm Hg & 13 & $(11,7)$ & 15 & $(9,7)$ & & 1,6 & $0,7-3,7$ \\
\hline HTA-2 $>160 />100 \mathrm{~mm} \mathrm{Hg}$ & 6 & $(5,4)$ & 2 & $(1,3)$ & $0,14^{\dagger}$ & 5 & $0,9-26$ \\
\hline \multicolumn{8}{|l|}{$\mathrm{IMC}\left(\mathrm{kg} / \mathrm{m}^{2}\right)$} \\
\hline Mediana (RIC) & 33,04 & $4(4,76)$ & 22,6 & $(2,72)$ & $0,00^{\star *}$ & & \\
\hline \multicolumn{8}{|l|}{ Perímetro braquial (cm) } \\
\hline Mediana (RIC) & 37,5 & $(5,5)$ & 29 & (5) & $0,00^{\star *}$ & & \\
\hline \multicolumn{8}{|l|}{ Perímetro de cintura $(\mathrm{cm})$} \\
\hline Mediana (RIC) & 105 & (14) & 81,6 & $(12,2)$ & $0,00^{\star *}$ & & \\
\hline \multicolumn{8}{|l|}{ Perímetro de cadera $(\mathrm{cm})$} \\
\hline Mediana (RIC) & 112 & $(12,5)$ & 90 & $(11,2)$ & $0,00^{\star *}$ & & \\
\hline \multicolumn{8}{|l|}{ Índice cintura-cadera } \\
\hline Mediana (RIC) & \multicolumn{2}{|r|}{$(0,08)$} & \multicolumn{2}{|c|}{$0,92(0,07)$} & $0,004^{\star *}$ & & \\
\hline \multicolumn{8}{|l|}{ Porcentaje de grasa corporal } \\
\hline Mediana (RIC) & \multicolumn{2}{|c|}{$31,4 \quad(18)$} & \multicolumn{2}{|c|}{$25,6(15)$} & $0,003^{\star *}$ & & \\
\hline \multicolumn{8}{|l|}{ Glucemia en ayunas (mg/dl) } \\
\hline Mediana (RIC) & 85 & $(15,5)$ & 83 & $(15)$ & $0,046^{\star *}$ & & \\
\hline \multicolumn{8}{|l|}{ Colesterol total (mg/dl) } \\
\hline Mediana (RIC) & 197 & (57) & 189 & (62) & 0,492 & & \\
\hline \multicolumn{8}{|l|}{ Mediana (RIC) } \\
\hline cLDL (mg/dl) & 41 & $(16)$ & 41 & $(15)$ & 0,887 & & \\
\hline \multicolumn{8}{|l|}{ Mediana (RIC) } \\
\hline VLDL (mg/dl) & 125 & $(49,8)$ & 117,8 & $(48,4)$ & 0,788 & & \\
\hline \multicolumn{8}{|l|}{ VLDL (mg/dl) } \\
\hline Mediana (RIQ) & 28,4 & $(22,6)$ & 24,9 & $(19,05)$ & 0,071 & & \\
\hline Triglicéridos (mg/dl) & & & & & & & \\
\hline Mediana (RIC) & 144 & (120) & 123 & (94) & $0,043^{\star *}$ & & \\
\hline Insulina (mUL) & & & & & & & \\
\hline Mediana (RIC) & 4,65 & $5(10,2)$ & 4,1 & $5(7,29)$ & 0,107 & & \\
\hline Hb "glucosilada" (\%) & & & & & & & \\
\hline Mediana (RIC) & 5,7 & $(0,9)$ & 5,4 & $(1)$ & $0,002^{* \neq}$ & & \\
\hline
\end{tabular}

IMC: índice de masa corporal; CHDL CLDL y VLDL: lipoproteínas de alta, baja y muy baja densidad, respectivamente; T_SIS_MED y T_DIAS_MED: tensión arterial sistólica y diastólica media, respectivamente ${ }^{*} p<0,05$

RIC: rango intercuartílico

$\dagger$ prueba de ji al cuadrado de Pearson

$¥$ prueba $\mathrm{U}$ de Mann-Whitney 
Cuadro 2. Frecuencias genotípicas y alélicas de los polimorfismos rs2167270 del gen LEP, rs1137101 del gen LEPR y rs 17782313 del gen MC4R

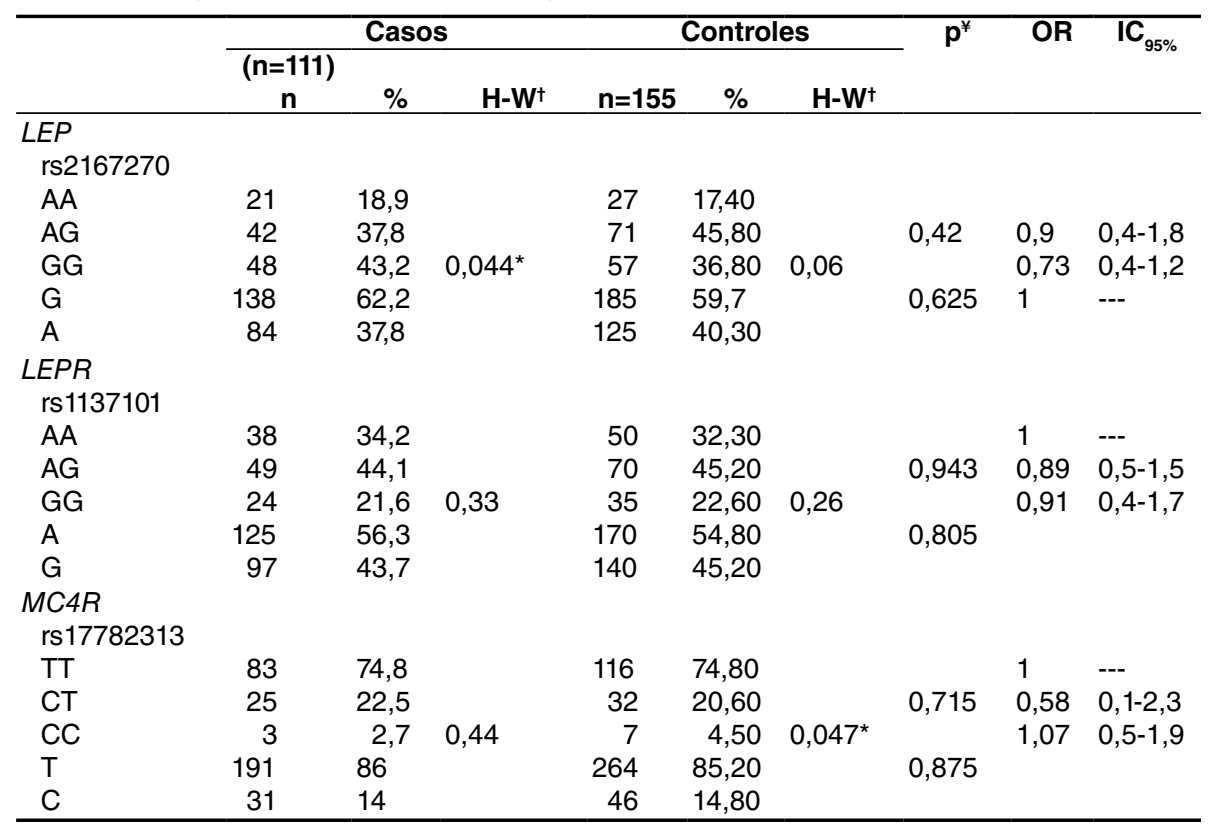

† test de equilibrio de Hardy-Weinberg mejorado con 100.000 pasos de la cadena Markov

¥ razón de verosimilitud de ji al cuadrado; OR $\left(\mathrm{IC}_{95 \%}\right)$ : modelos de regresión logística ajustados por sexo

Cuadro 3. Asociación de los polimorfismos rs2167270 del gen $L E P$, rs1137101 del gen $L E P R$ y rs17782313 del gen $M C 4 R$, con las variables clínicas y bioquímicas

\begin{tabular}{|c|c|c|c|c|c|c|c|c|c|c|c|c|c|}
\hline \multicolumn{14}{|l|}{ Grupo de casos } \\
\hline & \multicolumn{4}{|c|}{ LEP } & \multicolumn{4}{|c|}{ LEPR } & \multicolumn{5}{|c|}{ MC4R } \\
\hline & $\begin{array}{c}\text { AA }(n=21) \\
\text { Mediana (RIC) }\end{array}$ & $\begin{array}{c}\text { AG }(n=42) \\
\text { Mediana (RIC) }\end{array}$ & $\begin{array}{c}\text { GG }(n=48) \\
\text { Mediana (RIC) }\end{array}$ & $\begin{array}{c}\text { Valor } \\
p^{*}\end{array}$ & $\begin{array}{c}\text { AA }(n=38) \\
\text { Mediana (RIC) }\end{array}$ & $\begin{array}{c}\text { AG }(n=49) \\
\text { Mediana (RIC) }\end{array}$ & $\begin{array}{c}\text { GG }(n=24) \\
\text { Mediana (RIC) }\end{array}$ & $\begin{array}{c}\text { Valor } \\
p^{*}\end{array}$ & $\begin{array}{c}C C(n=3) \\
\text { Media (DE) }\end{array}$ & $\begin{array}{r}\text { CT ( } r \\
\text { Median }\end{array}$ & $\begin{array}{l}(n=25) \\
\text { na (RIC) }\end{array}$ & $\begin{array}{c}T T(n=83) \\
\text { Mediana (RIC) }\end{array}$ & $\mathbf{p}^{*}$ \\
\hline IMC [kg/m2] & $32,2 \quad[3,4]$ & $33,1 \quad[6,3]$ & $33,9 \quad[4,6]$ & 0,655 & $34,01[5,3]$ & $32,6[4,1]$ & $32,8 \quad[6,4]$ & 0,530 & $32,8[1,5]$ & 33,4 & {$[3,7]$} & $33,04 \quad[5,4]$ & 0,940 \\
\hline Índice cintura/cadera & $0,92[0,06]$ & $0,95[0,07]$ & $0,94[0,07]$ & 0,103 & $0,95[0,07]$ & $0,94[0,07]$ & $0,94[0,1]$ & 0,812 & $0,96[0,07]$ & 0,92 & {$[0,08]$} & $0,95[0,06]$ & 0,147 \\
\hline T_SIS_MED (mm Hg) & $113,3[21,5]$ & $117,6[25,5]$ & $119,8[20]$ & 0,853 & $118,6 \quad[14,5]$ & $119,6[20]$ & $115,5[35,1]$ & 0,967 & $128,1[12,9]$ & 110,6 & {$[18,3]$} & $120 \quad[24,6]$ & $0,025^{\star}$ \\
\hline T_DIAS_MED $(\mathrm{mm} \mathrm{Hg})$ & $71,3[18,6]$ & $72[16,7]$ & $75,5[14]$ & 0,724 & $76,5[16,8]$ & $72,6[13,5]$ & 69,5 [22] & 0,832 & $82[9,5]$ & 69,6 & {$[11,1]$} & $75,6[15,6]$ & 0,077 \\
\hline$\%$ grasa corporal & $28,1[15,7]$ & $37,6[16,5]$ & $30,3 \quad[17,6]$ & 0,089 & $32,1[16,6]$ & $29,7[18]$ & $35,6 \quad[19,5]$ & 0,845 & $24,5[8,9]$ & 29,7 & {$[19,5]$} & $31,7[16]$ & 0,409 \\
\hline Glucemia (mg/dl) & $82 \quad[24,7]$ & $87 \quad[16]$ & $84,5[14,5]$ & 0,570 & $83,8[11,5]$ & $83[20,5]$ & $88,5[22,5]$ & 0,077 & $87,5[8,2]$ & 82 & {$[16,5]$} & $86 \quad[16]$ & 0,234 \\
\hline $\mathrm{cHDL}(\mathrm{mg} / \mathrm{dl})$ & 42 [27] & $41[14,2]$ & {$[16]$} & 0,524 & $41 \quad[13,7]$ & 42 [17] & $41,5[14]$ & 0,640 & $43[14,7]$ & 49 & [18] & [15] & $0,048^{*}$ \\
\hline cLDL (mg/dl) & $112,8[39,6]$ & $127,3[51,5]$ & $127,1 \quad[53,4]$ & 0,393 & $113,1 \quad[56,5]$ & $124,8[50,4]$ & $133 \quad[39,4]$ & 0,252 & $103,4[22,1]$ & 127 & {$[47,8]$} & {$[57,2]$} & 0,554 \\
\hline VLDL (mg/dl) & $32,6[23]$ & $27,2[27,1]$ & $25 \quad[16,5]$ & 0,728 & $25,2[19,8]$ & $28,4[24,9]$ & $29,3[16,8]$ & 0,951 & $28,9[11,5]$ & 28,4 & {$[19,2]$} & $28,8 \quad[25,8]$ & 0,612 \\
\hline Triglicéridos (mg/dl) & $161[114]$ & 136 [132] & {$[91,7]$} & 0,616 & $123[122,3]$ & $146[116]$ & $146,5[112,5]$ & 0,861 & $154,9[59,4]$ & 145 & {$[96,5]$} & 141 [129] & 0,793 \\
\hline Insulina (mULO) & $5,02[9,4]$ & $3,98[9,1]$ & $6,64[11,5]$ & 0,742 & $4,76[11,7]$ & $5,52[9,95]$ & $3,60[11,07]$ & 0,963 & $15,3[13,4]$ & 7,9 & {$[11,5]$} & $3,96[9,2]$ & 0,157 \\
\hline $\mathrm{HbA1c}(\%)$ & $5,9 \quad[0,5]$ & $5,8 \quad[1,175]$ & $5,6 \quad[0,9]$ & 0,085 & $5,75[1,1]$ & $5,7 \quad[0,7]$ & $5,7 \quad[1,1]$ & 0,660 & $5,5[0,28]$ & 5,8 & {$[0,55]$} & $5,7 \quad[1]$ & 0,623 \\
\hline \multicolumn{14}{|l|}{ Grupo de control } \\
\hline & \multicolumn{4}{|c|}{ LEP } & \multicolumn{4}{|c|}{$\begin{array}{r}\text { LEPR } \\
\end{array}$} & \multicolumn{5}{|c|}{ MC4R } \\
\hline & $\begin{array}{c}\text { AA }(n=27) \\
\text { Mediana (RIC) }\end{array}$ & $\begin{array}{c}\text { AG }(n=71) \\
\text { Mediana (RIC) }\end{array}$ & $\begin{array}{c}\text { GG }(n=57) \\
\text { Mediana (RIC) }\end{array}$ & $\begin{array}{c}\text { Valor } \\
p^{*}\end{array}$ & $\begin{array}{c}\text { AA }(n=50) \\
\text { Mediana (RIC) }\end{array}$ & $\begin{array}{c}\text { AG }(\mathrm{n}=70) \\
\text { Mediana (RIC) }\end{array}$ & $\begin{array}{c}\mathrm{GG}(\mathrm{n}=35) \\
\text { Mediana (RIC) }\end{array}$ & \multirow[t]{2}{*}{$\begin{array}{c}\begin{array}{c}\text { Valor } \\
\mathbf{p}^{*}\end{array} \\
0151\end{array}$} & $\begin{array}{c}\text { CC }(n=7) \\
\text { Mediana (RIC) }\end{array}$ & \multicolumn{2}{|c|}{$\begin{array}{c}\text { CT }(n=32) \\
\text { Mediana (RIC) }\end{array}$} & $\begin{array}{c}\mathrm{TT}(\mathrm{n}=116) \\
\text { Mediana (RIC) }\end{array}$ & $\mathbf{p}^{*}$ \\
\hline IMC & $22,5 \quad(2,4)$ & $22,2 \quad(3,1)$ & 22,9 & 0,153 & 22,9 & $22,6 \quad(2,6)$ & $22,07 \quad(2,2)$ & & $24,06(2,01)$ & 22,3 & $(2,9)$ & 22,6 & 0,068 \\
\hline Índice cintura/cadera & $0,92(0,08)$ & $0,92(0,08)$ & $0,92(0,07)$ & 0,967 & $0,90(0,08)$ & $0,92(0,08)$ & $0,93 \quad(0,05)$ & 0,356 & $0,94(C$ & 0,90 & $(0,07)$ & $0,92(0,07)$ & 0,591 \\
\hline T_SIS_MED & $108,3(26,3)$ & $111,6(16,6)$ & $114,6(19,8)$ & 0,304 & $115 \quad(16,1)$ & $110,6(22,5)$ & $111,6 \quad(23)$ & 0,682 & $106,3(37)$ & 115,5 & $(25)$ & $112 \quad(16,2)$ & 0,738 \\
\hline T_DIAS_MED & $71,6(12,6)$ & $72,3(16,3)$ & $72 \quad(12,5)$ & 0,893 & $72,5 \quad(14,6)$ & $71,5(13,5)$ & $72,3 \quad(16,3)$ & 0,580 & $68,3(28,3)$ & 73,8 & $(14,7)$ & 71,8 & 0,303 \\
\hline$\%$ grasa corporal & $26,8(13,3)$ & $25,3(16,9)$ & $26,1 \quad(14,1)$ & 0,953 & $25,4(15,3)$ & $25,5(16,8)$ & $28,4 \quad(14,3)$ & 0,481 & 25,3 (15) & 23,05 & $(19,2)$ & $(14,1)$ & 0,685 \\
\hline Glucemia (mg/dl) & $82 \quad(15)$ & $84 \quad(15)$ & (16) & 0,832 & 84,5 (12) & $82(16,7)$ & (16) & 0,936 & $87(18)$ & 84,5 & $(15,7)$ & (15) & 0,263 \\
\hline Colesterol (mg/dl) & $183(49)$ & (56) & $(65,5)$ & 0,547 & $182 \quad(64,2)$ & $187,5(53,2)$ & (65) & 0,802 & $173(75)$ & 175,5 & $(64,5)$ & $(58,5)$ & 0,419 \\
\hline $\mathrm{cHDL}(\mathrm{mg} / \mathrm{dl})$ & $39 \quad(21)$ & (14) & $(15,5)$ & 0,561 & $(19,2)$ & $40,5(15)$ & (13) & 0,870 & $47(16)$ & 41,5 & $(17,2)$ & 40,5 & 0,716 \\
\hline $\mathrm{cLDL}(\mathrm{mg} / \mathrm{dl})$ & $112,4(36,5)$ & $115,6(45)$ & $(65,4)$ & 0,776 & $113,7 \quad(48,3)$ & $120 \quad(46,3)$ & $(64,8)$ & 0,756 & $114,8(38,2)$ & 106,4 & $(52,3)$ & $(52,8)$ & 0,391 \\
\hline VLDL (mg/dl) & $23,4(15,7)$ & $24,1(18,8)$ & (23) & 0,600 & $26 \quad(19,2)$ & $23,6(18,9)$ & $(21,6)$ & 0,425 & $22,2(10,8)$ & 26,4 & $(16,7)$ & $24,6 \quad(20,4)$ & 0,871 \\
\hline Triglicéridos (mg/dl) & $123 \quad(79)$ & 122 (97) & $(88,5)$ & 0,969 & 126,5 (102) & $114,5(93,2)$ & (97) & 0,421 & $111(54)$ & 131 & (70) & 121,5 (102) & 0,860 \\
\hline Insulina (mUL) & $4,97(7,1)$ & $3,58(7,08)$ & $3,91 \quad(7,6)$ & 0,398 & $4,3 \quad(7,3)$ & $4,55(6,3)$ & $3,51 \quad(9,6)$ & 0,753 & $5,56(8,1)$ & 2,5 & $(6,8)$ & $4,2 \quad(7,4)$ & 0,897 \\
\hline $\mathrm{HbA1c}$ (mg/dl) & $5,6 \quad(1,1)$ & $5,3(0,8)$ & $5,5 \quad(1,05)$ & 0,081 & $(0,95)$ & $5,3 \quad(1,1)$ & $5,4 \quad(1,1)$ & 0,828 & $5,1(1,3)$ & 5,2 & $(0,9)$ & $(0,97)$ & 0,432 \\
\hline
\end{tabular}
IMC: índice de masa corporal; cHDL cLDL y VLDL: lipoproteínas de alta, baja y muy baja densidad, respectivamente;T_SIS_MED y T_DIAS_MED: tensión arterial sistólica y diastólica media, respectivamente

${ }^{*} \mathrm{p}<0,05$

* prueba U de Mann-Whitney 


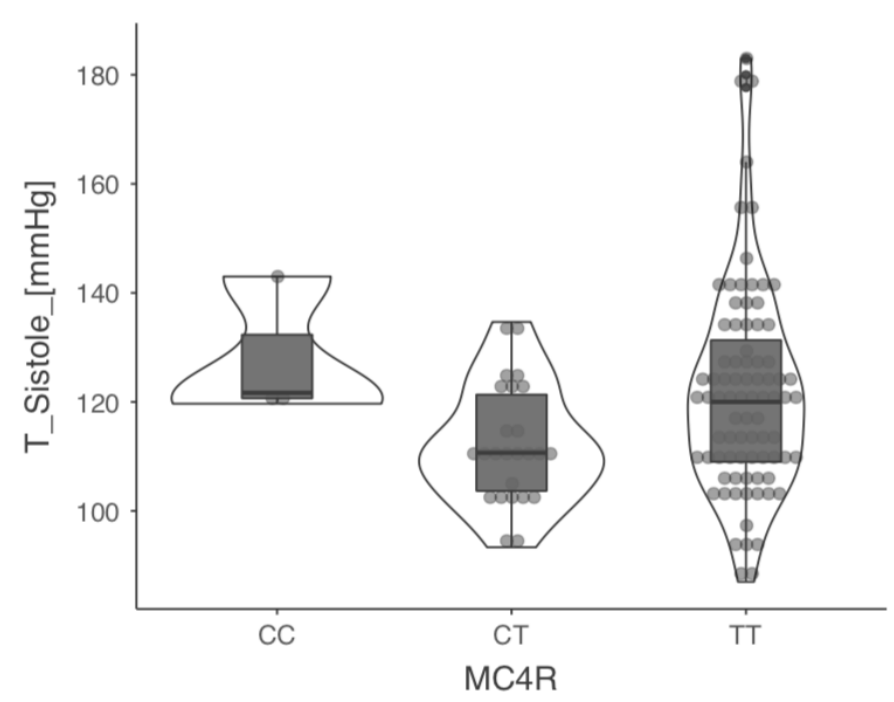

En los individuos obesos con genotipo CC, hubo un aumento de la tensión arterial sistólica promedio expresado como mediana de 128 (RIC: 12,9), a diferencia de los individuos con genotipos heterocigoto CT (mediana: 110,6; RIC: 18,3) y homocigoto TT (mediana: 120; RIC: 24,6; p: 0,025).

Figura 1. Asociación del polimorfismo rs 17782313 del gen $M C 4 R$ con la tensión arterial sistólica promedio en el grupo de adultos obesos

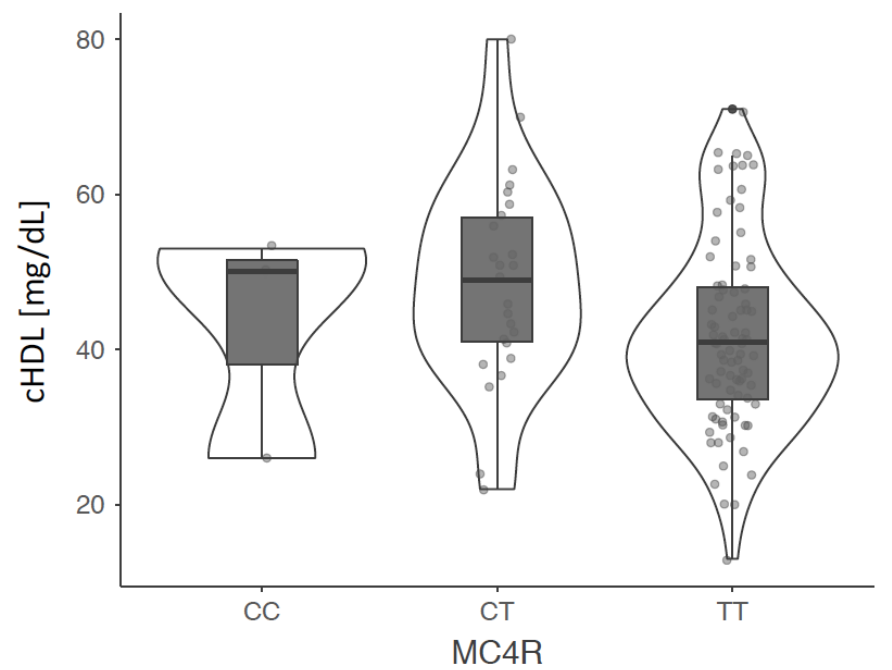

En los individuos obesos con genotipo TT hubo una disminución de las concentraciones séricas de HDL (mediana: 41; RIC: 15), a diferencia de los individuos con genotipos CC (mediana: 43; RIC: 14,7) y CT (mediana: 49; RIC: 18; p: 0,048).

Figura 2. Asociación del polimorfismo rs17782313 del gen MC4R con las concentraciones séricas de HDL en el grupo de adultos obesos

\section{Discusión}

Numerosos estudios se han enfocado en evaluar la presencia de algunos SNP en los genes de las proteínas que participan en el sistema leptinamelanocortina, para dilucidar la propensión genética a la obesidad con base en el papel de este sistema en la mantención del equilibrio entre el estímulo y la inhibición del apetito en función del gasto energético para controlar el peso corporal (17). En este sentido, en todo el mundo se han obtenido resultados contradictorios, ya que la asociación de estos polimorfismos con 
el IMC se ve influenciada por la etnia, la ubicación geográfica y los factores medioambientales; de ahí, la necesidad de estudiar la asociación de los polimorfismos rs2167270 del gen LEP, rs 1137101 del gen LEPR y rs17782313 del gen MC4R con la obesidad en una población colombiana.

Entre las variantes comunes del gen $L E P$, el polimorfismo rs2167270 ha sido uno de los más frecuentemente asociados con la obesidad. En el Family Heart Study, por ejemplo, un haplotipo común (49\%) que incluía este polimorfismo se asoció con un aumento del IMC en población de Estados Unidos (18). Asimismo, en un estudio de sujetos del Coronary Artery Risk Development in Young Adults Study (CARDIA), este polimorfismo mostró una asociación consistente con tres medidas antropométricas: el peso, el IMC y el perímetro de cintura de sujetos caucásicos (19). Además, Shruti, et al., encontraron una tendencia al aumento de los niveles del IMC y de leptina con cada adición del alelo A de la variante exónica rs2167270 en una población del sur de India (20). Sin embargo, en el presente estudio la frecuencia de este alelo A fue de $37,8 \%$ en el grupo de casos y de $40,3 \%$ en el grupo control, es decir, no tuvo significación estadística.

A pesar de que se ha reportado una fuerte asociación de este polimorfismo con la obesidad, no es posible sugerir una influencia directa de dicha variante en la expresión de la leptina y su función en la fisiopatología de la obesidad, ya que se encuentra dentro del primer exón no traducido del gen. Sin embargo, se puede sugerir que al estar en desequilibrio de ligamiento con el polimorfismo del promotor (rs7799039), rs2167270 puede tener un efecto sobre la transcripción del gen LEP (21).

En cuanto al polimorfismo rs 1137101 del gen $L E P R$, se ha reportado que su genotipo $G G$ se asoció con un menor riesgo de obesidad (OR=0,26; $\mathrm{IC}_{95 \%}$ $0,08-0,79)(p=0,018)$, pues redujo el IMC en $2,44 \mathrm{~kg} / \mathrm{m}^{2}$ en individuos del sur de Chile (22). Asimismo, Chavarría-Ávila, et al., han señalado que el alelo G podría ser un marcador genético asociado con una menor acumulación de grasa corporal en la población del oeste mexicano (23). En este sentido, la frecuencia del alelo $G$ en nuestra población fue predominante $(45,2 \%)$ en el grupo con peso normal ( $p>0,05)$, lo cual es similar a lo encontrado en poblaciones de Chile y México. Por su parte, el genotipo AA se asoció significativamente con la obesidad en voluntarios de Túnez (OR=1,41; IC ${ }_{95 \%}$ $1,035-1,85)(p=0,045)(24)$.

En el presente estudio, la frecuencia de este genotipo predominó en el grupo de obesos $(p>0,05)$. Por el contrario, De Oliveira, et al., reportaron una fuerte asociación del genotipo GG con el exceso de peso en población brasilera $\left(\mathrm{OR}=2,14, \mathrm{IC}_{95 \%}, 1,01-4,52\right)(\mathrm{p}=0,047)(25)$, al igual que Mărginean, et al., quienes concluyeron que los sujetos portadores de los genotipos $G G$ o AG tenían 3,06 veces más riesgo de desarrollar obesidad en comparación con los portadores de $A A\left(O R=3,06 ; I_{95 \%} 1,70-5,51\right)(p=0,0001)(26)$.

Estas discrepancias de los polimorfismos de LEP y LEPR también se evidenciaron en un metaanálisis del 2015 de Ghalandari, et al. (27). De los diecisiete artículos revisados, nueve informaban sobre una asociación significativa, o los consideraban como posibles factores de riesgo; sin embargo, no se encontró esta relación en los ocho estudios restantes, que incluían, entre otras, poblaciones de Brasil y México con resultados similares a los del presente estudio. 
Lo mismo sucede con el polimorfismo rs 17782313 del gen $M C 4 R$, el cual se ha asociado con un elevado IMC $(8,28,29)$ y con la aparición temprana de obesidad grave (9). Se ha sugerido que los portadores del genotipo TT de este polimorfismo tienen un nivel notablemente mayor de hipermetilación en el promotor de MC4R en comparación con los portadores de los genotipos CT y CC, lo que puede contribuir a regular la expresión de $M C 4 R$ y, por consiguiente, al desarrollo de la obesidad (30). Sin embargo, en otros estudios no se ha podido demostrar la asociación de este polimorfismo con la obesidad, como es el caso de las investigaciones en población polaca y africana $(8,31)$. En este sentido, un hallazgo notable del presente estudio es que el genotipo TT fue igual en los casos y los controles.

En resumen, el análisis genético del presente estudio, comparado con los de otras poblaciones, evidenció la falta de asociación de los polimorfismos con la obesidad, resultados que se explicarían porque las poblaciones están expuestas a diferentes factores medioambientales o de estilo de vida, y ello es fundamental para determinar la influencia de cada polimorfismo en la obesidad. Se hace necesario analizar la interacción entre polimorfismo y ambiente para aclarar la contribución de estas variantes genéticas al desarrollo de la obesidad.

Así como en este estudio, otros investigadores han evaluado la influencia de estos polimorfismos en las variables clínicas y bioquímicas relacionadas con la obesidad. En cuanto al perfil de lipoproteínas, se pudo demostrar que los portadores del alelo $\mathrm{G}$ del polimorfismo rs 1137101 del gen LEPR tenían niveles más bajos de triglicéridos y lipoproteínas de muy baja densidad (VLDL) en el sur de Chile (22), y niveles más elevados de colesterol total, pero más reducidos de cHDL en sujetos de Túnez (32). Por su parte, el alelo $\mathrm{C}$ del polimorfismo rs 17782313 del gen MC4R se ha asociado con una disminución de la lipoproteína antiaterogénica en mujeres italianas con diabetes gestacional y mujeres sanas de China $(33,34)$, resultado similar al reportado en escolares mexicanos $\left(\mathrm{OR}=2,99, \mathrm{IC}_{95 \%} 1,93-4,64\right)(\mathrm{p}<0,0001)$. Por el contrario, en el presente estudio se encontró una asociación estadística del alelo $\mathrm{T}$ y el genotipo homocigoto de este polimorfismo con una disminución del cHDL en el grupo de casos.

En cuanto a las variables relacionadas con el metabolismo de la glucosa, Yang, et al., informaron que el polimorfismo rs1137101 del gen LEPR se asoció con cifras elevadas de HbA1C (34), y Boumaiza, et al., demostraron su asociación con un aumento de glucemia basal e insulinemia en voluntarios de Túnez (32). Asimismo, Garcés, et al., concluyeron en su estudio que el genotipo A/A de este polimorfismo confería a los niños obesos que lo portaban 2,6 veces más riesgo de desarrollar resistencia a la insulina que aquellos con los genotipos G/G y A/G (35). Además, se pudo demostrar que esta variante genética puede considerarse como un factor de riesgo significativo para la diabetes mellitus de tipo II en sujetos de Malasia (36).

Estos polimorfismos también se han asociado con la presión arterial, pero no se ha encontrado significación estadística en dicha relación en algunas poblaciones. Así, Fan, et al., no encontraron una asociación del polimorfismo rs2167270 del gen LEP en una población multiétnica de Malasia (37), así como tampoco se observó la influencia de rs17782313 del gen MC4R en la presión arterial de mujeres chinas (34). En contraste, el alelo A del polimorfismo rs1137101 del gen LEPR se asoció con una elevación de la presión arterial sistólica (37) en sujetos brasileros hipertensos, en quienes se evidenció que los valores fueron significativamente menores en los hombres 
portadores de genotipos con, al menos, una copia del alelo $\mathrm{C}$ de rs17782313 del gen MC4R (38). En concordancia con estos resultados, el análisis genético del presente estudio demostró que el genotipo $C C$ del polimorfismo rs17782313 del gen MC4R se asoció con un aumento de la presión arterial sistólica en el grupo de obesos.

Aunque los mecanismos que vinculan la obesidad con la hipertensión no han sido completamente aclarados, se sabe que el aumento de la actividad simpática del sistema nervioso central contribuye a la elevación de la presión arterial en los sujetos obesos. La evidencia indica que la leptina y el sistema de melanocortina, incluidos los receptores 4 de la melanocortina (MC4R), juegan un papel clave en la relación de la obesidad con el aumento de la actividad del sistema nervioso central y la hipertensión, lo cual representa un objetivo importante para el tratamiento de la obesidad y sus consecuencias metabólicas y cardiovasculares (39).

Este trabajo se vio limitado por el tamaño de la muestra, por lo que se recomienda hacer estudios con una muestra más grande que permita un análisis estadístico con mayor poder.

En conclusión, los polimorfismos rs2167270 del gen LEP, rs1137101 del gen LEPR y rs17782313 del gen MC4R no se asociaron con la obesidad en la población analizada. El polimorfismo rs 17782313 del gen MC4R fue el único que se asoció con el aumento de la presión arterial sistólica y con la disminución del cHDL en sujetos con obesidad.

\section{Referencias}

1. Organización Mundial de La Salud. Obesidad y sobrepeso. Nota descriptiva - 2018. Fecha de consulta: 25 de julio de 2018. Disponible en: http://www.who.int/es/news-room/fact-sheets/ detail/obesity-and-overweight

2. Schwartz MW, Woods SC, Porte D, Seeley RJ, Baskin DG. Central nervous system control of food intake. Nature. 2000;404:661-71. https://doi.org/10.1038/35007534

3. Farooqi IS, O'Rahilly S. Leptin: A pivotal regulator of human energy homeostasis. Am J Clin Nutr. 2009;89:980S-4. https://doi.org/10.3945/ajcn.2008.26788C

4. Santos J. Sistema leptina-melanocortinas en la regulación de la ingesta y el peso corporal. Rev Med Chile. 2009;137:1225-34. http://doi.org/10.4067/S0034-98872009000900014

5. Wauman J, Tavernier J. Leptin receptor signaling: Pathways to leptin resistance. Front Biosci (Landmark Ed). 2011;17:2771-93.

6. Dasgupta S, Salman M, Siddalingaiah LB, Lakshmi G, Xaviour D, Sreenath J. Genetic variants in leptin: Determinants of obesity and leptin levels in South Indian population. Adipocyte. 2015;4:135-40. https://doi.org/10.4161/21623945.2014.975538

7. Hassan N, El-Masry S, Zarouk W, El Banna R, Mosaad R, Al-Tohamy M, et al. Obesity phenotype in relation to gene polymorphism among samples of Egyptian children and their mothers. Genes Dis. 2017;5:150-7. https://doi.org/10.1016/j.gendis.2017.12.004

8. Xi B, Chandak GR, Shen Y, Wang Q, Zhou D. Association between common polymorphism near the MC4R gene and obesity risk: A systematic review and meta-analysis. PLoS One. 2012;7:e45731. https://doi.org/10.1371/journal.pone.0045731

9. Meyre D, Delplanque J, Chèvre JC, Lecoeur C, Lobbens S, Gallina S, et al. Genome-wide association study for early-onset and morbid adult obesity identifies three new risk loci in European populations. Nat Genet. 2009;41:157-9. https://doi.org/10.1038/ng.301

10. Giraldo-Castrillón Y, Muñoz F, Navarro-Lechuga E, Segura-Cardona Á. Factores de riesgo para disfunción sistólica ventricular izquierda en adultos de un programa de salud global. Rev Cuid. 2017;8:1519-28. https://doi.org/10.15649/cuidarte.v8i1.371

11. Mattevi VS, Zembrzuski VM, Hutz MH. Association analysis of genes involved in the leptinsignaling pathway with obesity in Brazil. Int J Obes. 2002;26:1179-85. http://doi.org/10.1038/sj.ijo.0802067 
12. World Health Organization. Obesity, preventing and managing the global epidemic-report of a WHO consultation on obesity - 1997. Fecha de consulta: 14 de octubre de 2018. Disponible en: https://www.who.int/nutrition/publications/obesity/WHO TRS 894/en/

13. Organización Mundial de la Salud. Información general sobre la hipertensión arterial en el mundo - 2013. Fecha de consulta: 14 de octubre de 2018. Disponible en: https://apps.who.int/ iris/bitstream/handle/10665/87679/WHO_DCO_WHD_2013.2_spa.pdf;jsessionid=87D07D18 DA6FA31420743611C3BE7176? sequence $=1$

14. James PA, Oparil S, Carter BL, Cushman WC, Dennison-Himmelfarb C, Handler J, et al. 2014 evidence-based guideline for the management of high blood pressure in adults: Report from the panel members appointed to the Eighth Joint National Committee (JNC 8). JAMA. 2014;311:507-20. https://doi.org/10.1001/jama.2013.284427

15. Fridewald WT, Levin RY, Fredrickson DS. Estimations of the concentration of c-LDL in plasma without use of the preparative ultracentrifuge. Clin Chem. 1972;18:499-507.

16. National Cholesterol Education Program (NCEP) Expert Panel on Detection, Evaluation, and Treatment of High Blood Cholesterol in Adults (Adult Treatment Panel III). Third Report of the National Cholesterol Education Program (NCEP) Expert Panel on Detection, Evaluation, and Treatment of High Blood Cholesterol in Adults (Adult Treatment Panel III) final report. Circulation. 2002;106:3143-421.

17. González E, Aguilar MJ, Padilla C, García I. Obesidad monogénica humana: papel del sistema leptina-melanocortina en la regulación de la ingesta de alimentos y el peso corporal en humanos. Anales Sis San Navarra. 2012;35:285-93. https://doi.org/10.4321/S113766272012000200010

18. Jiang Y, Wilk JB, Borecki I, Williamson S, DeStefano AL, Xu G, et al. Common variants in the 5 ' region of the leptin gene are associated with body mass index in men from the National Heart, Lung, and Blood Institute Family Heart Study. Am J Human Genet. 2004;75:220-30. https://doi.org/10.1086/422699

19. Friedlander Y, Li G, Fornage M, Williams O, Lewis C, Schreiner C, et al. Candidate genes from molecular pathways related to appetite regulatory neural network and adipocyte homeostasis and obesity: The Coronary Artery Risk Development in Young Adults (CARDIA) Study. Ann Hum Genet. 2010;74:387-98. https://doi.org/10.1111/j.14691809.2010.00596.x

20. Dasgupta S, Salman M, Siddalingaiah LB, Lakshmi G, Xaviour D, Sreenath J. Genetic variants in leptin: Determinants of obesity and leptin levels in South Indian population. Adipocyte. 2015;4:135-40. https://doi.org/10.4161/21623945.2014.975538

21. Li WD, Reed DR, Lee JH, Xu W, Kilker RL, Sodam BR, et al. Sequence variants in the 5' flanking region of the leptin gene are associated with obesity in women. Ann Hum Genet. 1999;63:227-34. https://doi.org/10.1046/j.1469-1809.1999.6330227.x

22. Manríquez V, Avilés J, Salazar L, Saavedra N, Serón P, Lanas F, et al. Polymorphisms in genes involved in the leptin-melanocortin pathway are associated with obesity-related cardiometabolic alterations in a Southern Chilean population. Mol Diagn Ther. 2018;22:10113. https://doi.org/10.1007/s40291-017-0306-8

23. Chavarría-Ávila E, Vázquez-Del Mercado M, Gómez-Bañuelos E, Ruiz-Quezada SL, CastroAlbarrán J, Sánchez-López L, et al. The impact of LEPG-2548A and LEPR GIn223Arg polymorphisms on adiposity, leptin, and leptin-receptor serum levels in a Mexican Mestizo population - 2015. Biomed Res Int. 2015;2015:539408. https://doi.org/10.1155/2015/539408

24. Nesrine Z, Haithem H, Imen B, Fadoua N, Asma O, Fadhel N, et al. Leptin and leptin receptor polymorphisms, plasma leptin levels and obesity in Tunisian volunteers. Int J Exp Path. 2018;99:121-30. https://doi.org/10.1111/iep.12271

25. De Oliveira R, Cerda A, Dalla F, Feraz M, Armaganijan D, Silveira M, et al. Leptin receptor gene polymorphisms are associated with adiposity and metabolic alterations in Brazilian individuals. Arq Bras Endocrinol Metabol. 2013;57:677-84. https://doi.org/10.1590/S000427302013000900002

26. Mărginean CO, Mărginean $\mathrm{C}$, Voidăzan $\mathrm{S}$, Meliţ L, Crauciuc $\mathrm{A}$, Duicuet $\mathrm{C}$, et al. Correlations between leptin gene polymorphisms 223 A/G, 1019 G/A, 492 G/C, 976 C/A, and anthropometrical and biochemical parameters in children with obesity. A prospective case-control study in a Romanian population-The Nutrichild Study. Medicine (Baltimore). 2016;95:e3115. https://doi.org/10.1097/MD.0000000000003115

27. Ghalandari H, Hosseini-Esfahani F, Mirmiran P. The association of polymorphisms in leptin/ leptin receptor genes and ghrelin/ghrelin receptor genes with overweight/obesity and the related metabolic disturbances: A review. Int J Endocrinol Metab. 2015;13:e19073. https://doi.org/10.5812/ijem.19073v2 
28. García-Solís P, Reyes-Bastidas M, Flores K, García O, Rosado J, Méndez-Villa L, et al. Fat mass obesity-associated (FTO) (rs9939609) and melanocortin 4 receptor (MC4R) (rs17782313) SNP are positively associated with obesity and blood pressure in Mexican school-aged children. Br J Nutr. 2016;116:1834-40. https://doi.org/10.1017/S0007114516003779

29. Almeida SM, Furtado JM, Mascarenhas P, Ferraz M, Ferreira J, Monteiro M, et al. Association between LEPR, FTO, MC4R, and PPARG-2 polymorphisms with obesity traits and metabolic phenotypes in school-aged children. Endocrine. 2018;60:466-78. https://doi.org/10.1007/s12020-018-1587-3

30. Tang Y, Jin B, Zhou L, Lu W. MeQTL analysis of childhood obesity links epigenetics with a risk SNP rs17782313 near MC4R from meta-analysis. Oncotarget. 2017;8:2800-6. https://doi.org/10.18632/oncotarget.13742

31. Leońska-Duniec A, Jastrzębski Z, Zarębska A, Smółka W, Cięszczyk P. Impact of the polymorphism near MC4R (rs17782313) on obesity- and metabolic-related traits in women participating in an aerobic training program. J Hum Kinet. 2017;58:111-9. https://doi.org/10.1515/hukin-2017-0073

32. Boumaiza I, Omezzine A, Rejeb J, Rebhi L, Ben Rejeb N, Nabli N, et al. Association between four resistin polymorphisms, obesity, and metabolic syndrome parameters in Tunisian volunteers. Genet Test Mol Biomarkers. 2012;16:1356-62. https://doi.org/10.1089/gtmb.2012.0156

33. Franzago M, Fraticelli F, Nicolucci A, Celentano C, Liberati M, Stuppia L, et al. Molecular analysis of a genetic variants panel related to nutrients and metabolism: association with susceptibility to gestational diabetes and cardiometabolic risk in affected women. J Diabetes Res. 2017:2017:4612623. https://doi.org/10.1155/2017/4612623

34. Yang J, Gao Q, Gao X, Tao X, Cai H, Fan Y, et al. Melanocortin-4 receptor rs17782313 polymorphisms are associated with serum triglycerides in older Chinese women. Asia Pac J Clin Nutr. 2016;25:213-9. https://doi.org/10.6133/apjcn.2016.25.1.18

35. Garcés MF, Gómes B, Stekman H, Hernández C, López A, Soto de Sanabria I. Polimorfismos G2548A del gen de leptina y GLN223ARG del gen del receptor de leptina en pre-púberes con riesgo cardiometabólico. Arch Venez Puer Ped. 2016;79:54-61.

36. Etemad A, Ramachandran V, Pishva SR, Heidari F, Aziz A, Yusof A, et al. Analysis of GIn223Agr polymorphism of leptin receptor gene in type II diabetic mellitus subjects among Malaysians. Int J Mol Sci. 2013;14:19230-44. https://doi.org/10.3390/ijms140919230

37. Fan S-H, Say $\mathrm{Y}-\mathrm{H}$. Leptin and leptin receptor gene polymorphisms and their association with plasma leptin levels and obesity in a multi-ethnic Malaysian suburban population. J Physiol Anthropol. 2014;33:15. https://doi.org/10.1186/1880-6805-33-15

38. Marcadenti A, Fuchs FD, Matte U, Sperb F, Moreira LB, Fuchs SC. Effects of FTO RS9939906 and MC4R RS17782313 on obesity, type 2 diabetes mellitus and blood pressure in patients with hypertension. Cardiovasc Diabetol. 2013;12:103. https://doi.org/10.1186/1475-2840-12-103

39. Do Carmo JM, da Silva AA, Dubinion J, Sessums PO, Ebaady S, Wang Z, et al. Control of metabolic and cardiovascular function by the leptin-brain melanocortin pathway. IUBMB Life. 2013;65:692-8. https://doi.org/10.1002/iub.1187 